\title{
CONTRADIÇÕES NO COMÉRCIO DE MADEIRA CERTIFICADA EM UMA REGIÃO METROPOLITANA DO NORDESTE DO BRASIL
}

\author{
Douglas Marques Silva ${ }^{1}$, Marlyete Chagas de $\mathrm{Araújo}^{2}$, Victor Farias Silva ${ }^{1}$, Enrico Bernard ${ }^{3}$ \\ ${ }^{1}$ Acadêmico de Ciências Biológicas, UFPE, Recife, PE, Brasil - douglasmarques.bio@gmail.com; vtr.fariasqui@hotmail.com \\ ${ }^{2}$ Acadêmica de Ciências Biológicas, UFPE, Recife, PE, Brasil - marlyete.chagas@ hotmail.com \\ ${ }^{3}$ Biólogo, Dr., Depto. de Zoologia, UFPE, Recife, PE, Brasil - enrico.bernard@ufpe.br \\ Recebido para publicação: 22/05/2013 - Aceito para publicação: 27/03/2014
}

\begin{abstract}
Resumo
A exploração ilegal de madeira é forte agente de desmatamento, comprometendo biodiversidade e serviços ambientais. A certificação florestal é uma forma de oposição ao mercado ilegal de madeira, e vendedores e compradores responsáveis e bem informados são fundamentais no comércio dessa madeira. O comércio de madeira certificada na Região Metropolitana de Recife, Pernambuco, foi investigado de forma a 1) avaliar o conhecimento dos vendedores sobre a certificação de produtos madeireiros, 2) acessar o estado do mercado local e 3) identificar dificuldades e perspectivas sobre o consumo desse tipo de produto na região. Em entrevistas em 33 empresas, foi identificado que o comércio local de madeira certificada é marcado por contradições. Cerca de $75 \%$ dos vendedores não sabem indicar corretamente o produto certificado e quase metade desconhece os selos e as informações de identificação. De maneira geral, vendedores não dominam conceitos, marcos legais e instrumentos de certificação. Embora declarem-se sensíveis ao impacto da extração madeireira, as empresas pouco se engajam em projetos ambientais ou campanhas sobre o uso de madeira certificada. Apesar das contradições, há um ambiente favorável para a disseminação da certificação e comercialização desses produtos, mas ajustes - sobretudo no fluxo de informação - são necessários nesse processo. Palavras-chave: Certificação florestal; Conselho Brasileiro de Manejo Florestal; FSC.
\end{abstract}

\begin{abstract}
Contradictions in the certified wood trade in a metropolitan area in northeastern Brazil. Illegal timber extraction is an important driver of deforestation, compromising biodiversity and its environmental services. Certification is a strategy to oppose illegal market, and responsible and wellinformed sellers and buyers are crucial players. Here, the trade of certified wood in the Metropolitan Area of Recife, Pernambuco, is investigated in order to a) evaluate the level of knowledge from local sellers on the certification process, b) access the status of the certified market, and c) identify difficulties and perspectives for certified timber in the region. Based on 33 interviews, we identified that the local certified wood trade is marked by contradictions. About $75 \%$ of salespeople do not know how to correctly indicate a certified product, and almost half ignore certification seals and how to correctly identify certified wood. In general, sellers are unaware of basic certification concepts, legal frameworks and certification protocols. Although declaring some environmental concern, selling companies are rarely engaged in environmental projects or campaigns on the use of certified wood. Despite the contradictions, there is a favorable environment for the spread of the certification concept and marketing of these products, but adjustments - especially on the information flow - are necessary in this process.

Keywords: Conselho Brasileiro de Manejo Florestal; forest certification; FSC.
\end{abstract}

\section{INTRODUÇÃO}

Estimativas apontam que cerca da metade da exploração madeireira do sudeste Asiático, África Central, Rússia e América do Sul, e entre $64 \%$ e $80 \%$ da produção madeireira da Amazônia brasileira são ilegais (MILLER et al., 2006). A exploração ilegal de madeira é uma atividade complexa, com várias ramificações e associações com outros setores ilegais (KISHOR, 2012), mas é inequivocamente apontada como um forte agente de desmatamento de florestas tropicais e tem sérias consequências para a 
manutenção da biodiversidade e dos serviços ambientais por ela prestados (VAN SCHAICK et al., 2001; FEARNSIDE, 2005; GUTIERREZ-VELEZ; MACDICKEN， 2008; CLARK; COVEY, 2012; SHEARMAN et al., 2012).

O comércio ilegal de madeira pressiona de forma desleal aqueles que operam na legalidade e desincentiva o cumprimento das leis vigentes (MILLER et al., 2006; KISHOR, 2012). Por ser um mercado bastante heterogêneo, vários mecanismos de enfrentamento são necessários para a solução desse problema. Como oposição ao mercado ilegal de madeira, diversas iniciativas de mercado para certificação e identificação de produtos de origem legal e controlada surgiram nos últimos 30 anos (AULD et al., 2008). Essas iniciativas buscam a padronização de processos e produtos e a ampliação de um cenário de governança em um setor que opera largamente na ilegalidade (DIETER, 2009). O conceito de certificação florestal é relativamente simples: um produtor adota excelência na exploração madeireira de modo a satisfazer padrões técnicos, ambientais e sociais preestabelecidos, recebe um selo de aprovação emitido por certificadoras independentes e ganha assim acesso a um mercado de consumidores dispostos a pagar preços mais altos pela exploração sustentável da madeira (SCHULZE et al., 2008). São exemplos os selos emitidos pelo Forest Stewardship Council (FSC) ou pelo Programa Brasileiro de Certificação Florestal (CERFLOR). De maneira geral, a certificação tem sido apontada como positiva do ponto de vista ambiental, social e econômico (DAMETTE; DELACOTE, 2011; MOORE et al., 2012; TIKINA et al., 2012; BASSO et al., 2012) e áreas de florestas certificadas ao redor do planeta têm crescido. Em janeiro de 2013, o Brasil tinha cerca de 7,2 milhões de hectares de florestas certificadas somente pelo FSC (FSC BRASIL, 2013).

Vendedores e compradores responsáveis e bem informados são parte fundamental do comércio de produtos certificados e tais iniciativas podem falhar pela falta de conhecimento, engajamento ou interesse das pessoas envolvidas na parte final do processo, a comercialização dos bens (AGUILAR; VLOSKY, 2007; ARAÚJO et al., 2009; PEATIE, 2010). Estudos sobre produção e certificação de madeira são relativamente abundantes (AULD et al., 2008; PUTZ et al., 2012), mas, de maneira contraditória, poucos estudos abordam a parte final da cadeia de custódia da madeira certificada. Neste estudo, investigamos o comércio de madeira certificada na Região Metropolitana de Recife, Pernambuco, uma importante metrópole do Nordeste do Brasil, com foco nos vendedores de madeira. A Região Metropolitana de Recife experimenta um intenso processo de crescimento comercial e industrial, com um aumento da demanda de madeira tanto pela construção civil quanto para atender as diversas indústrias que se instalam ou passam por processo de ampliação na região. Dessa forma, os objetivos principais foram 1) avaliar o conhecimento dos vendedores sobre a certificação de produtos madeireiros, 2) acessar o estado do mercado local e 3) identificar dificuldades e perspectivas sobre o consumo desse tipo de produto na região.

\section{MATERIAL E MÉTODOS}

A Região Metropolitana de Recife (RMR - $\left.8^{\circ} 04^{\prime} 03^{\prime \prime} \mathrm{S}, 34^{\circ} 55^{\prime} 00^{\prime \prime} \mathrm{W}\right)$ tem 14 municípios, em uma área de cerca de $2800 \mathrm{~km}^{2}$, com uma população aproximada de 3,6 milhões de habitantes e renda per capita de $\mathrm{R} \$ 12.250,38$ (IBGE, 2010). A partir de lista telefônica impressa e on line, 72 madeireiras na RMR foram identificadas e contatadas por telefone, para verificar aquelas em atividade. Sessenta empresas estavam ativas e 33 aceitaram participar da pesquisa, sendo visitadas ao longo de 21 dias de novembro de 2011. Em cada uma, o responsável pelo estabelecimento foi entrevistado, utilizando um formulário com 25 perguntas, para a caracterização da empresa, perfil do consumidor, tipo, volume, origem da madeira comercializada e aspectos do comércio de madeira certificada. Para algumas dessas perguntas, o entrevistado tinha a opção de apontar mais de uma resposta.

As madeireiras foram classificadas como microempresas (faturamento anual igual ou menor a $\mathrm{R} \$ 240.000,00$ ), médias (entre $\mathrm{R} \$ 240.000,00$ e $\mathrm{R} \$ 12.000 .000,00$ ) ou grandes empresas (faturamento maior que $\mathrm{R} \$ 12.000 .000,00)$. O comprador foi categorizado em pessoa física ou jurídica. Para verificar se os entrevistados tinham conhecimento correto das diversas formas de comercialização de madeiras (p. ex., legal, certificada, ilegal), eles foram solicitados a apontar, dentre múltiplas respostas, aquela que melhor correspondia à definição de madeira certificada. Foram perguntados ainda sobre as fontes de informação quanto à certificação florestal, sobre qual tipo de madeira comercializavam, procedência e, quando comercializando madeira certificada, quais as variedades, quais tipos de selos utilizados pela 
empresa e qual o fator motivador para a oferta desse tipo de produto. Outras perguntas foram sobre a importância da comercialização de madeira certificada, se a empresa desenvolvia alguma estratégia específica para a publicidade da certificação florestal e sobre o envolvimento em programas de recuperação e/ou reflorestamento de áreas impactadas por extração de madeira. As perguntas finais eram sobre a frequência de fiscalização, sobre qual a melhor maneira de o comprador poder tomar ciência da certificação no momento da compra, sobre a demanda e dificuldades no comércio desse tipo de produto e sobre expectativas futuras. Para essas perguntas, havia a possibilidade de respostas em aberto, para a ampliação do leque de respostas. Os dados foram tabulados em planilha eletrônica Excel e analisados com estatística descritiva básica.

\section{RESULTADOS}

Das 33 madeireiras entrevistadas, 63,6\% declararam-se de microporte, 33,3\% de médio porte e apenas uma empresa de grande porte. Quanto ao perfil do consumidor, $42,4 \%$ das empresas declararam atender principalmente pessoas físicas, $39,4 \%$ a pessoas jurídicas e 18,2\% a ambas. Quanto à procedência da madeira, de 63 origens declaradas, 53,9\% dos entrevistados afirmaram ser a Região Norte o principal fornecedor, e o Pará $(39,7 \%)$ foi o estado mais frequente nas respostas.

Oitenta e oito por cento dos entrevistados afirmaram conhecer o conceito de madeira certificada. Embora a maior parte dos entrevistados $(82,8 \%)$ tenha associado madeira certificada à existência de plano de manejo (Figura 1A), apenas 27,6\% indicaram corretamente a necessidade de certificação atribuída por órgãos específicos. Oito fontes de informação sobre certificação madeireira foram citadas (Figura 2A), sendo a televisão $(32,4 \%)$ e a internet $(20,6 \%)$ as mais citadas.

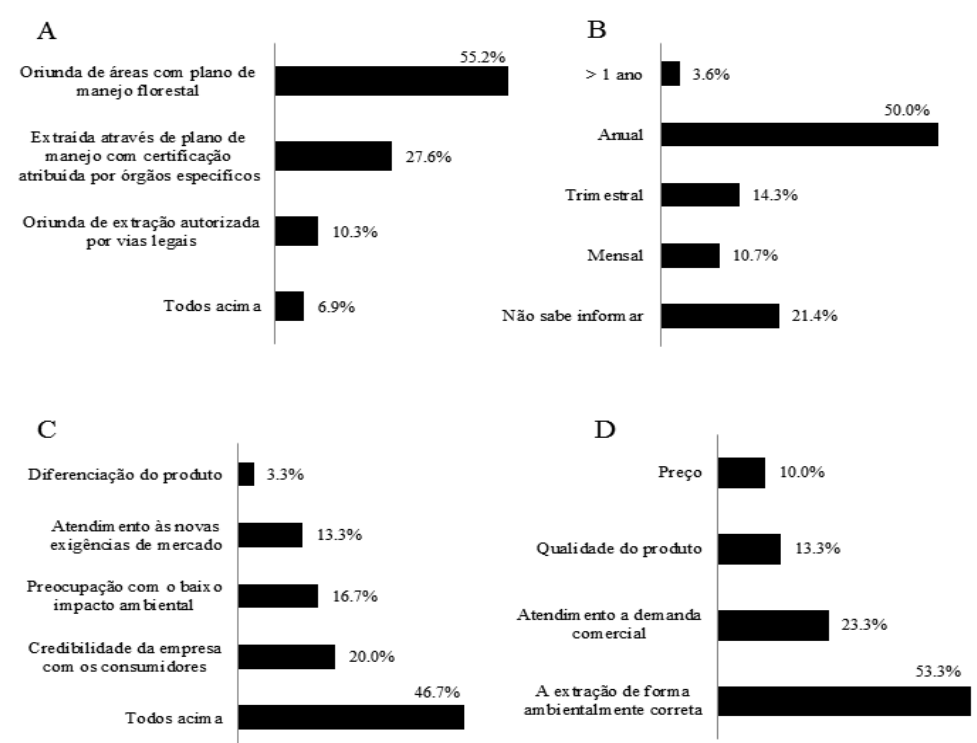

Figura 1. Frequência de respostas obtida em 33 entrevistas com vendedores de madeira na Região Metropolitana de Recife, Pernambuco, em novembro de 2011. As perguntas referiam-se à definição de madeira certificada (A), à frequência de fiscalização sobre suas empresas (B), à importância do uso de madeira certificada $(\mathrm{C})$ e às razões pelas quais a empresa vende madeira certificada (D).

Figure 1. Frequency of answers obtained in 33 interviews with wood sellers in the Metropolitan Area of Recife, Pernambuco, in November 2011. Questions related to the definition of certified wood (A), the frequency of inspection over their business (B), the importance of using certified wood $(\mathrm{C})$, and the reasons why the company sells certified wood (D).

Quanto ao tipo de madeira, $87,9 \%$ dos entrevistados afirmaram vender madeira legal e certificada (Figura 2B). Entre os 30 informantes que declararam vender madeira certificada, $46,7 \%$ não 
sabiam qual o selo utilizado. Para os 53,3\% que declararam saber o selo de certificação, os mais indicados foram o FSC (16,7\%) e o CERFLOR (13,3\%) (Figura 2C). Sete entrevistados apontaram outro tipo de selo e, entre estes sete, 85,7\% responderam possuir o selo "DOF" (Documento de Origem Florestal) e um dos entrevistados apontou o "GTIN" (Global Trade Item Number, ou Número Global de Item Comercial) como documentos de certificação, apesar de nenhum dos dois se tratar de selo de certificação propriamente dito.
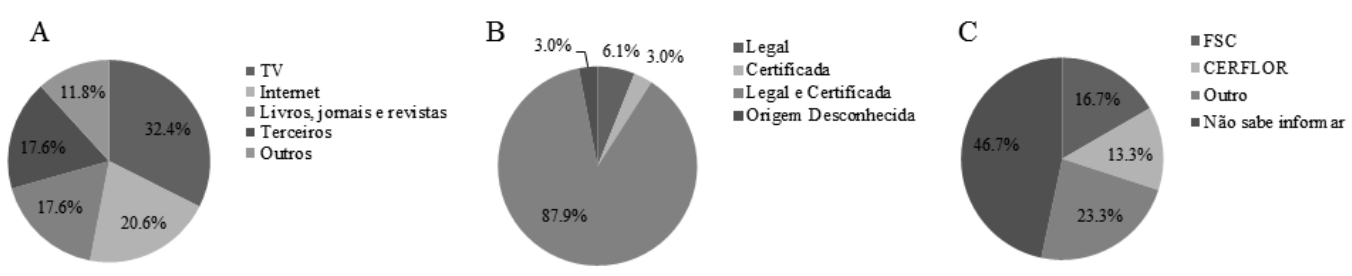

Figura 2. Frequência de respostas obtida em 33 entrevistas com vendedores de madeira na Região Metropolitana de Recife, Pernambuco, em novembro de 2011. As perguntas referiam-se às fontes de informação sobre madeira certificada (A), ao tipo de madeira vendida pela empresa (B) e, em caso de venda de madeira certificada, ao selo de certificação utilizado (C).

Figure 2. Frequency of answers obtained from 33 interviews with wood sellers in the Metropolitan Area of Recife, Pernambuco, in November 2011. Questions related to the sources of information on certified wood (A), the type of wood sold by the companies (B) and, in case of sale of certified wood, on which certification seal used (C).

Pouco mais da metade dos entrevistados $(51,5 \%)$ indicou vender menos que 10 espécies de madeira, 33,3\% de 10 a 20 espécies, $6,0 \%$ acima dessa quantidade e $9,1 \%$ não souberam responder a essa pergunta. "Eucalipto", "jatobá" e "angelim-pedra" foram as três variedades mais citadas. Dos 30 entrevistados que disseram saber a quantidade de espécies comercializada, 56,7\% afirmaram que menos de 10 espécies são certificadas, 30,0\% indicaram entre 10 e 20 espécies certificadas e um entrevistado afirmou comercializar mais de 20 espécies certificadas.

Quanto ao volume, 45,4\% das empresas declararam que adquirem menos de $100 \mathrm{~m}^{3}$ de madeira/mês, $33,3 \%$ de 100 a $1000 \mathrm{~m}^{3} /$ mês e $21,2 \%$ desconhecem o volume comercializado/mês. Dentre os 29 tipos de madeiras vendidas, sobressaem o eucalipto (14,3\%) e o jatobá (12,6\%). Mais de $60 \%$ dos entrevistados declararam que acima de $76 \%$ da madeira comercializada possui selo de certificação.

A maior parte dos entrevistados $(84,8 \%)$ afirmou haver fiscalização frequente da madeira vendida e, deste total, 50,0\% apontaram frequência anual (Figura 1B). Apenas um entrevistado afirmou que a fiscalização ocorre em intervalo maior que um ano. Quando indagados sobre como o consumidor poderia se certificar que está comprando madeira certificada, $36,4 \%$ dos 33 entrevistados não souberam responder à pergunta, 27,3\% apontaram a presença de selo de certificação na embalagem ou no produto, $15,1 \%$ apontaram o Documento de Origem Florestal, 9,1\% indicaram a nota fiscal como prova ao consumidor, $6,1 \%$ indicaram o ato de perguntar na hora da compra, um $(3,0 \%)$ disse que a resposta seria dada pela fornecedora da empresa e um $(3,0 \%)$ afirmou que não havia como o comprador saber.

Dos 30 entrevistados que declararam vender madeira certificada, 53,3\% apontaram a extração de forma ambientalmente correta como o principal motivo para a venda desse tipo de produto (Figura 1D), superando o atendimento à demanda comercial, a qualidade do produto e o preço. Quando perguntados sobre a importância da venda desse tipo de produto, 20,0\% dos entrevistados declararam que comercializam madeira certificada porque isso aumenta a credibilidade da empresa junto ao mercado consumidor (Figura 1C), superando as preocupações com o impacto ambiental da extração madeireira, o atendimento às exigências de mercado e a diferenciação de seu produto junto ao mercado. Quase metade dos entrevistados apontou todas as alternativas anteriores (Figura 1C). Entretanto, apenas três $(9,1 \%)$ dos 33 entrevistados apontaram que suas empresas desenvolvem ou apoiam algum projeto relacionado à recuperação e/ou reflorestamento de áreas impactadas por extração de madeira, e apenas cinco empresas 
$(15,1 \%)$ afirmaram desenvolver algum tipo de campanha sobre a importância do uso de madeira certificada.

Quanto ao mercado de madeira certificada na região, $69,7 \%$ dos entrevistados afirmaram haver uma demanda crescente, apoiada em um aumento da procura por esse tipo de madeira. A maior parte dos entrevistados $(72,7 \%)$ indicou não experimentar problemas na aquisição de madeira certificada e 75,7\% afirmaram não encontrar dificuldades no seu comércio. Dos nove entrevistados que indicaram experimentar problemas de aquisição, o preço inviável (33,3\%), a má divulgação sobre como adquirir $(33,3 \%)$ e a alta burocracia para adquirir $(33,3 \%)$ foram apontados como os maiores empecilhos.

Quanto ao futuro do mercado de madeira certificada na região, $81,8 \%$ possuem expectativas positivas, apontando uma maior preocupação do comprador tanto em relação à qualidade do produto quanto a fatores ambientais da extração. Os vendedores também esperam que a fiscalização e a legislação sobre o assunto sejam mais rígidas no futuro, resultando em um combate mais forte ao comércio ilegal da madeira. Entretanto, $12,1 \%$ dos entrevistados indicaram certo receio quanto ao futuro desse mercado, devido a falhas de fiscalização da certificação, bem como no aumento da burocracia nesse processo. Dois entrevistados $(6,1 \%)$ não possuem expectativas formadas sobre o futuro do mercado.

\section{DISCUSSÃO}

Este estudo mostra que o comércio de madeira certificada na Região Metropolitana do Recife (RMR) tem várias contradições. Embora a maioria dos vendedores de madeira da região afirme conhecer tanto o conceito de madeira certificada quanto vender madeira legal e certificada, cerca de $75 \%$ dos vendedores não sabem como identificar e indicar corretamente um produto certificado. Menos de 1/3 dos entrevistados indicaram a necessidade de certificação atribuída por órgãos específicos, e quase metade desconhece os selos usados na certificação e as informações de identificação desse tipo de madeira. Alguns apontam como atestados de certificação documentos incorretos (nota fiscal, Documento de Origem Florestal ou o Global Trade Information Number). Um vendedor chegou a afirmar que o consumidor simplesmente não tem como saber se a madeira que está comprando é ou não certificada. Tais fatos mostram que a maior parte dos vendedores de madeira na RMR não domina conceitos, marcos legais e instrumentos de verificação de certificação, e existe a clara necessidade da melhoria da comunicação entre certificadoras, fornecedores certificados e vendedores de madeira na RMR.

Se a certificação florestal não é totalmente conhecida pelos vendedores, o processo de informação sobre a disponibilidade e as vantagens desses produtos para os compradores também não será feito de forma adequada no momento da compra. Isso representa uma falha de comunicação que pode comprometer a difusão de produtos certificados (PEATTIE, 2010). Mais além, a televisão foi o meio de informação mais importante para a maior parte dos entrevistados, reforçando a falta de um canal oficial de informação sobre certificação madeireira. Além dos esforços de educação do consumidor sobre o processo de certificação e suas características, as certificadoras e certificados também devem redobrar esforços para esclarecer junto aos vendedores da madeira sobre quais os selos disponíveis no mercado, e como a madeira certificada pode e deve ser corretamente identificada e separada da não certificada (MILLER et al., 2006). A separação inicial de madeira ilegal e legal, e a mensagem de que só é certificada aquela com selo de certificação específico, devem ficar claras entre todos os envolvidos na cadeia de custódia da madeira. Como constatado, na RMR essa mensagem ainda não atingiu esse estágio.

Foi constatado que, para os vendedores, o mais determinante para o uso de madeira certificada é a forma de extração ambientalmente correta. Novamente uma contradição: embora declarem-se sensíveis à questão do impacto ambiental da extração de madeira, quase não existe envolvimento de quem vende a madeira em projetos de reflorestamento e/ou recuperação de áreas degradadas. Além disso, apenas 15\% dos vendedores afirmaram fazer algum tipo de campanha sobre o uso de madeira certificada. A mensagem de preocupação ambiental dessas empresas parece incompleta: mostram-se solidários à redução dos impactos da extração madeireira, mas poucos fazem além de solidarizarem-se.

O mercado doméstico em pequena escala é frequentemente negligenciado quando do combate ao comércio ilegal de madeira, mas é apontado como essencial contra a ilegalidade (LAWSON; MACFAUL, 2010; KISHOR, 2012). As madeireiras situadas na RMR são, em geral, de microporte, atendendo principalmente pessoas físicas. Isso indica que certificadores e certificados, se interessados em melhorar a informação sobre a certificação, terão que lidar com um número maior de pessoas. Difundir eficientemente informações corretas sobre a certificação é um desafio para certificadores e certificados, e 
resta saber se estes se interessarão por um mercado de pequeno porte, em detrimento de grandes compradores e vendedores. Sem esse interesse, a maior parte do mercado de madeira da RMR não se beneficiará do uso de madeira certificada e de suas consequências socioambientais.

As madeireiras pesquisadas vendem madeira legal e certificada, embora em quantidade limitada, para atender a uma pequena, porém crescente, demanda na região. A maior parte dos entrevistados tem uma expectativa positiva quanto ao comércio de madeira certificada, devido à preocupação crescente com a qualidade do produto e com fatores ambientais, e afirma não encontrar problemas para a aquisição ou venda de madeira certificada. Essa expectativa positiva é uma oportunidade: o setor é receptivo à madeira certificada, reconhece suas características diferenciadas e parece apostar que esse mercado tende a crescer. Tal cenário é favorável à certificação, e o setor tem a chance de dialogar com um público receptivo à causa, facilitando possíveis mudanças de hábitos e costumes (PEATTIE, 2010).

Para os entrevistados que indicaram dificuldade na obtenção de madeira certificada, o preço mais elevado, a alta burocracia para adquiri-la e a má difusão do procedimento de certificação foram apontados como os principais empecilhos. A procura por produtos ambientalmente corretos vem mudando com os anos (ESPACH, 2006; AULD, 2008), está em alta no mercado atual, e a demanda por essa madeira vem valorizando o produto. É justo que um produto diferenciado e com maior valor agregado, como a madeira certificada, tenha preços acima da madeira convencional e da ilegal (ESPACH, 2006). Equalizar os custos de produção, a oferta e a demanda por essa madeira é uma tarefa complexa. Entretanto, é necessária atenção especial para que o preço diferenciado da madeira certificada não acabe afastando os interessados pelo produto.

O preço da madeira certificada sofre com concorrência desleal da madeira ilegal, e a fiscalização é crucial no combate ao desmatamento e ao comércio clandestino de madeira. Com mais frequência, a fiscalização contribui para que responsáveis pela extração ilegal sejam punidos e, principalmente, para que a demanda por madeira ilegal decresça (MILLER et al., 2006). As madeireiras da RMR indicaram que recebem a visita de fiscais pelo menos uma vez ao ano. Embora haja fiscalização, a frequência dessas visitas é baixa. Nossa pesquisa mostra que os próprios vendedores esperam que a fiscalização e a legislação sejam mais rígidas em um futuro, resultando em um combate mais forte ao comércio ilegal da madeira. A maior parte da madeira vendida na RMR tem origem amazônica, e sua principal fonte, o estado do Pará, aumenta o risco de clandestinidade, uma vez que esse estado é historicamente associado à exploração madeireira ilegal (MONTEIRO et al., 2011; MONTEIRO et al., 2012). A real origem da madeira consumida na RMR deve passar por maior escrutínio, e a melhor verificação de procedência deve ser incentivada. Desde abril de 2006, Recife e Olinda fazem parte da iniciativa "Cidade Amiga da Amazônia", do Greenpeace, cujo objetivo é incentivar prefeituras brasileiras a adotarem leis que evitem o consumo de madeira nativa de origem criminosa nas compras e licitações públicas. Tal iniciativa deu origem à Lei $\mathrm{n}^{\mathrm{o}} 17.433$, de 19 de março de 2008, que autoriza a política de fomento à utilização de madeira certificada no município de Recife. Embora superficial, iniciativas como esta são positivas e devem ser replicadas.

Embora tenha sido mostrado que o comércio de madeira certificada na Região Metropolitana de Recife é marcado por contradições, reconhece-se que a certificação total desse mercado é um objetivo que deve ser buscado e estimulado, pois os benefícios ambientais, sociais e econômicos a ele associados são necessários. Os resultados aqui apresentados mostram que ajustes - sobretudo no fluxo de informação são necessários nesse processo, mas há um ambiente favorável para uma melhor disseminação do conceito de certificação e comercialização desses produtos, e essa oportunidade não deve ser desperdiçada.

\section{CONCLUSÕES}

- O comércio de madeira certificada na Região Metropolitana de Recife, Pernambuco, é marcado por contradições entre o discurso e a prática.

- De maneira geral, vendedores não dominam conceitos, marcos legais e instrumentos de certificação, e embora declarem-se sensíveis ao impacto da extração de madeira, as empresas pouco se engajam em projetos ambientais ou campanhas sobre o uso de madeira certificada.

- Apesar das contradições, há um ambiente favorável para a disseminação do conceito de certificação e comercialização desses produtos, mas ajustes - sobretudo no fluxo de informação - são necessários nesse processo. 


\section{AGRADECIMENTOS}

A todos os entrevistados, por permitirem a realização desta pesquisa e disponibilizarem seu tempo para a obtenção das informações necessárias. Aos graduandos Carolina Cabral, Lyssandra Teti e Luis Felipe Silva (UFPE), pelo auxílio na coleta de dados. Esta pesquisa é fruto da disciplina Metodologia Científica, ministrada no curso de Bacharelado em Ciências Biológicas com ênfase em Ciências Ambientais - CCB UFPE, ao qual somos gratos.

\section{REFERÊNCIAS}

AGUILAR, F, X.; VLOSK, R. P. Consumer willingness to pay price premiums for environmentally certified wood products in the U.S. Forest Policy and Economics, v. 9, n. 8, p. 1100 - 1112, 2007.

ARAÚJO, M.; KANT, S.; COUTO, L. Why Brazilian companies are certifying their forests? Forest Policy and Economics, v. 11, n. 8, p. 579 - 585, 2009.

AULD, G.; GULBRANDSEN, L. H.; MCDERMOTT, C. L. Certification schemes and the impacts on forests and forestry. Annu. Rev. Environment Resources, v. 33, p. 187 - 211, 2008.

BASSO, V. M.; JACOVINE, L. A. G.; ALVES, R. R.; NARDELLI, A. M. B. Contribuição da certificação florestal ao atendimento da legislação ambiental e social no estado de Minas Gerais. Revista Árvore, v. 36, n. 4 , p. $747-757,2012$.

CLARK, J. A.; COVEY, K. R. Tree species richness and the logging of natural forests: A meta-analysis. Forest Ecology and Management, v. 276, p. 146 - 153, 2012.

DAMETTE, O.; DELACOTE, P. Unsustainable timber harvesting, deforestation and the role of certification. Ecological Economics, v. 70, p. 1211 - 1219, 2011.

DIETER, M. Analysis of trade in illegally harvested timber: Accounting for trade via third party countries. Forest Policy and Economics, v. 11, n. 8, p. 600 - 607, 2009.

ESPACH, R. When is sustainable forestry sustainable? The Forest Stewardship Council in Argentina and Brazil. Global Environmental Politics, v. 6, p. 55 - 84, 2006.

FEARNSIDE, P. Deforestation in Brazilian Amazonia: History, rates, and consequences. Conservation Biology, v. 19, n. 3, p. 680 - 688, 2005.

FOREST STEWARDSHIP COUNCIL (FSC BRASIL). Disponível em: <http://br.fsc.org/>. Acesso em: fev. 2013

GUTIERREZ-VELEZ, V. H.; MACDICKEN, K. Quantifying the direct social and governmental costs of illegal logging in the Bolivian, Brazilian, and Peruvian Amazon. Forest Policy and Economics, v. 10, p. $248-256,2008$.

INSTITUTO NACIONAL DE METROLOGIA, QUALIDADE E SERVIÇO (INMETRO). Normas Brasileiras Cerflor. Disponível em <http://www.inmetro.gov.br/qualidade/cerflor_normasBrasileiras. asp>. Acesso em: fev. 2013.

KISHOR, N. Controlling illegal logging in domestic and international markets by harnessing multi-level governance opportunities. International Journal of the Commons, v. 6, n. 2, p. 255 - 270, 2012.

LAWSON, S.; MACFAUL, L. Illegal Logging and Related Trade: Indicators of the Global Response. London: Chatham House, 2010. Disponível em: <http://www.chathamhouse.org.uk/publications/papers/ view/-/id/911/>. Acesso em: fev. 2013.

MILLER, F.; TAYLLOR, R.; WHITE, G. World Wildlife Fund - WWF. Seja Legal - Boas práticas para manter a madeira ilegal fora de seus negócios, 2006. Disponível em: <http://assets.wwf.org.br/ downloads/seja_legal_baixa.pdf>. Acesso em: fev. 2013. 
MONTEIRO, A.; CARDOSO, D.; CONRADO, D.; VERÍSSIMO, A.; SOUZA JR., C. Boletim Transparência Manejo Florestal Estado do Pará (2010-2011). Belém: Imazon, p. 16, 2012. Disponível em: <http://www.imazon.org.br/publicacoes/transparencia-manejo-florestal/boletim-transparencia-manejoflorestal-estado-do-para-2010-a-2011>. Acesso em: fev. 2013.

MONTEIRO, A.; CARDOSO, D.; CONRADO, D.; VERÍSSIMO, A.; SOUZA JR., C. Boletim Transparência Manejo Florestal Estado do Pará (2009 e 2010). Belém: Imazon, p. 16, 2011. Disponível em: <http://www.imazon.org.br/publicacoes/transparencia-manejo-florestal/boletim-transparencia-manejoflorestal-do-mato-grosso-2009-a-2010>. Acesso em: fev. 2013.

MOORE, S. E.; CUBBAGE, F.; EICHELDINGER, C. Impacts of Forest Stewardship Council (FSC) and Sustainable Forestry Initiative (SFI) Forest Certification in North America. J. Forestry, v. 110, n. 2, p. 79 - 88, 2012.

PEATTIE, K. Green consumption: Behavior and norms. Annu. Rev. Environ. Resour, v. 35, p. 195 $228,2010$.

PROGRAMA BRASILEIRO DE CERTIFICAÇÃO FLORESTAL (CERFLOR). Disponível em: <www.inmetro.gov.br/qualidade/cerflor_normasBrasileiras.asp> Acesso em: fev. 2013.

PUTZ, F. E.; ZUIDEMA, P. A.; SYNNOTT, T.; PEÑA-CLAROS, M.; PINARD, M. A.; SHEIL, D.; VANCLAY, J. K.; SIST, P.; GOURLET-FLEURY, S.; GRISCOM, B.; PALMER, J.; ZAGT, R. Sustaining conservation values in selectively logged tropical forests: the attained and the attainable. Conservation Letters, v. 5, n. 4, p. 296 - 303, 2012.

SCHULZE, M.; GROGAN, J.; VIDAL, E. Forest certification in Amazonia: standards matter. Oryx, v. 42, n. 2, p. 229, 2008.

SHEARMAN, P.; BRYAN, J.; LAWRANCE, W. F. Are we approaching 'peak timber' in the tropics? Biological Conservation, v. 151, p. 17 - 21, 2012.

VAN SCHAICK, C. P.;MONK, K. A.; ROBERTSON, M. Y. Dramatic decline in orang-utan numbers in the Leusser Ecosystem, Northern Sumatra. Oryx, v. 35, n. 1, p. 14 - 25, 2001.

TIKINA, A. V.; KOZAK, R. A.; INNES, J. L.; DUINKER, P. N.; LARSON, B. C. Forest certification in Canada: An exploratory study of perceptions of provincial and territorial government employees. Forestry Chronicle, v. 88, n. 1, p. 40 - 48, 2012. 